\title{
Moteurs piézo-électriques à onde progressive : I. Modélisation de la conversion d'énergie mécanique à l'interface stator/rotor
}

\author{
P. Minotti $\left({ }^{*}\right)$, P. Le Moal, L. Buchaillot et A. Ferreira

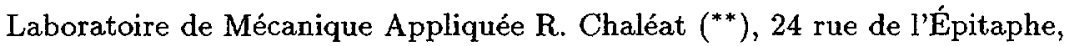 \\ 25030 Besançon, France
}

(Reçu le 3 avril 1996, accepté le 3 juillet 1996)

PACS.43.40.At - Experimental and theorical studies of vibrating systems

PACS.84.60.Bk - Performance characteristics of energy conversion systems; figure of merit

\begin{abstract}
Résumé. - La modélisation des moteurs piézo-électriques à onde progressive implique une grande variété de phénomènes physiques et mécaniques. Cette variété conduit à des approches et modèles tout aussi nombreux et variés, qui reposent principalement sur des analyses phénoménologiques et numériques (Méthode Élements Finis), et ne permettent pas de répondre aux exigences actuelles concernant le développement d'outils C.A.O. performants. Cette nécessité nous a conduits à développer une modélisation théorique analytique de la conversion d'énergie à l'interface stator/rotor. Ce papier est le premier d'une série de trois articles consacrés à la modélisation des moteurs piézo-électriques rotatifs. Après une rapide description des principes de fonctionnement de ces piézomoteurs, les hypothèses mécaniques et tribologiques concernant le mécanisme d'entraînement du rotor sont énoncées succinctement. On démontre ensuite que la modélisation cinématique et dynamique du stator, combinée à une représentation statique du comportement à l'interface stator/rotor, autorise l'évaluation des caractéristiques en charge des moteurs à onde progressive. Enfin, le logiciel baptisé C.A.S.I.M.M.I.R.E., récemment développé sur la base de la modélisation mécanique précédente, est présenté puis testé. Dans le dernier article de cette série, nous confirmerons la validité des simulations théoriques issues de ce logiciel, à partir de la caractérisation expérimentale de moteurs japonais de la firme SHINSEI. Ce nouveau logiciel constitue d'ores et déjà un outil performant en vue de l'optimisation des futurs moteurs à onde progressive, et a déjà fait l'objet d'une première exploitation en milieu industriel.
\end{abstract}

\begin{abstract}
The modeling of traveling wave type piezoelectric motors involves a large variety of mechanical and physical phenomena and therefore leads to numerous approaches and models. The latter, mainly based on phenomenological and numerical (based on Finite Element Method) analyses, are not suitable for current objectives oriented toward the development of efficient C.A.D. tools. As a result, an attempt is done to investigate analytical approaches, in order to theoretically model the mechanical energy conversion at the stator/rotor interface. This paper is the first in a serie of three articles devoted to the modeling of such rotative motors. After a short description of the operating principles specific to the piezomotors, the mechanical and tribological assumptions made for the driving mechanism of the rotor are briefly described. Then it is shown that the kinematic and dynamic modeling of the stator, combined with the static representation of the stator/rotor interface, gives an efficient way in order to perform the calculation of the loading characteristics of the driving shaft. Finally, the specifications of a
\end{abstract}

$\left({ }^{*}\right)$ Auteur auquel doit être adressée la correspondance

$\left({ }^{* *}\right)$ URA 004 C.N.R.S.

(C) Les Éditions de Physique 1996 
new software named C.A.S.I.M.M.I.R.E., which has been recently developed on the basis of our earlier mechanical modeling, are described. In the last of these three papers, the theoretical simulations performed on SHINSEI Japanese motors will show to be close to the experimental data and that the results reported in this paper will lead to the structural optimization of future traveling wave ultrasonic motors.

\section{Notations}

\begin{tabular}{|c|c|c|}
\hline$E_{1}, E_{2}, E_{3}$ & $\begin{array}{l}\text { modules d'Young du stator, du rotor (substrat), et de } \\
\text { la couche de friction }\end{array}$ & $\mathrm{MPa}$ \\
\hline$\nu_{1}, \nu_{2}, \nu_{3}$ & $\begin{array}{l}\text { coefficients de Poisson du stator, du rotor (substrat), et } \\
\text { de la couche de friction }\end{array}$ & \\
\hline$h_{1}, h_{2}, h_{3}$ & $\begin{array}{l}\text { épaisseurs du stator, du rotor (substrat), et de la } \\
\text { couche de friction }\end{array}$ & $\mathrm{m}$ \\
\hline$G_{2}, G_{3}$ & $\begin{array}{l}\text { transformées de Fourier des fonctions d'Airy en } \\
\text { contraintes du rotor (substrat), et de la couche de friction }\end{array}$ & \\
\hline$P$ & point matériel situé à la surface du stator & \\
\hline $\mathbf{u}_{\mathrm{p}}\left(u_{\mathrm{r}}, u_{\mathrm{q}}, u_{\mathrm{z}}\right)$ & vecteur déplacement du point $\mathrm{P}$ & $\mathrm{m}$ \\
\hline$\left(\mathbf{e}_{r}, \mathbf{e}_{\theta}, \mathbf{e}_{z}\right)$ & base orthonormale liée au stator & \\
\hline$U_{\theta}, U_{\mathrm{z}}$ & $\begin{array}{l}\text { amplitudes crêtes des déplacements dans les directions } \\
\theta \text { et } z\end{array}$ & $\mathrm{~m}$ \\
\hline$w, w^{(1)}, w^{(2)}$ & $\begin{array}{l}\text { vibrations hors-plan correspondant respectivement à } \\
\text { l'onde progressive et aux deux ondes stationnaires }\end{array}$ & $\mathrm{m}$ \\
\hline$\rho_{1}$ & masse volumique du matériau constitutif $d u$ stator & $\mathrm{kg} \mathrm{m}-3$ \\
\hline$H_{1}$ & distance plan moyen - surface du stator & $\mathrm{m}$ \\
\hline$k, n$ & nombre de diamètres et de cercles de nœuds & \\
\hline$\xi_{k n}$ & fonction de Bessel & \\
\hline$\omega_{k n}$ & $\begin{array}{l}\text { pulsation propre du stator associée aux formes propres } \\
\text { de rang }(k, n)\end{array}$ & $\operatorname{rad~} \mathrm{s}^{-1}$ \\
\hline$\omega$ & pulsation des signaux électriques. & $\operatorname{rad~s} s^{-1}$ \\
\hline$\lambda$ & $\begin{array}{l}\text { longueur d'onde de la vibration mécanique suivant la } \\
\text { direction } \theta\end{array}$ & $\mathrm{m}$ \\
\hline$\Lambda$ & facteur d'ellipse & \\
\hline$A$ & $\begin{array}{l}\text { amplitude de la vibration mécanique au rayon extérieur } \\
\left(r=R_{1}\right)\end{array}$ & $\mathrm{m}$ \\
\hline$\vec{A}$ & $\begin{array}{l}\text { amplitude moyenne dans la direction radiale de la } \\
\text { vibration mécanique }\end{array}$ & $\mathrm{m}$ \\
\hline$\left(v_{2}, w_{2}\right),\left(v_{3}, w_{3}\right)$ & $\begin{array}{l}\text { déplacements suivant } \theta \text { et } z \text { de points matériels appartenant } \\
\text { respectivement au substrat (2) et à la couche de friction ( } 3 \text { ) }\end{array}$ & $\mathrm{m}$ \\
\hline$w_{2 \mathrm{f}}$ & $\begin{array}{l}\text { déplacement hors-plan dû aux effets de flexion de points } \\
\text { matériels appartenant au substrat }\end{array}$ & $\mathrm{m}$ \\
\hline$K^{*}$ & rigidité en flexion du substrat & $\mathrm{MPa} \mathrm{m}$ \\
\hline$R_{0}, R_{1}$ & $\begin{array}{l}\text { rayons intérieur et extérieur de l'interface de contact } \\
\text { rotor/stator }\end{array}$ & $\mathrm{m}$ \\
\hline$p^{(1)}, . ., p^{(n)}$ & discrétisation de la distribution de pression $p(\theta)$ & $\mathrm{MPa}$ \\
\hline$R_{z}(\theta)$ & interpolation polynomiale de la distribution de pression $p(\theta)$ & $\mathrm{Nm}^{-1}$ \\
\hline
\end{tabular}




\begin{tabular}{|c|c|c|}
\hline$p_{\mathrm{s}}$ & pression statique à l'interface rotor/stator & $\mathrm{MPa}$ \\
\hline$F_{\text {ext }}$ & effort de précontrainte & $\mathrm{N}$ \\
\hline$\Gamma_{\mathrm{r}}, \Gamma, \Gamma_{\mathrm{s}}$ & couple résistant, couple moteur et couple statique & $N \mathrm{~m}$ \\
\hline$\Omega_{\mathrm{v}}, \Omega_{\mathrm{c}}$ & $\begin{array}{l}\text { vitesse angulaire de l'arbre moteur respectivement à } \\
\text { vide et en charge }\end{array}$ & $\operatorname{rad~} \mathrm{s}^{-1}$ \\
\hline$V_{\text {glis }}$ & vitesse de glissement des particules statoriques & $\mathrm{m} \mathrm{s}^{-1}$ \\
\hline$P_{\text {glis }}$ & pertes mécaniques à l'interface rotor/stator & W \\
\hline$K, b$ & taux de contact et borne de la zone de contact & $\%, \mathrm{~m}$ \\
\hline $\bar{b}$ & $\begin{array}{l}\text { abscisse circonférentielle de la particule statorique dont la } \\
\text { vitesse s'identifie à celle communiquée à l'arbre moteur : } \bar{V}_{\theta}\end{array}$ & $\mathrm{m}$ \\
\hline $\bar{b}_{\mathrm{c}}$ & lieu de $\bar{b}$ en fonction du couple résistant & $\mathrm{m}$ \\
\hline$\mu_{\mathrm{s}}, \mu_{\mathrm{d}}$ & $\begin{array}{l}\text { coefficient de frottement statique et dynamique à } \\
\text { l'interface rotor/stator }\end{array}$ & \\
\hline
\end{tabular}

\section{Introduction}

Les performances toujours plus exigeantes imposées aux machines modernes confinent les moteurs électromagnétiques conventionnels à leurs limites extrêmes de fonctionnement. Dans ce contexte, le développement d'un nouveau type de vibromoteurs résonnants, reposant sur le principe de la conversion d'une vibration mécanique (souvent ultrasonore) du stator, en un mouvement discontinu ou continu du rotor, présente un intérêt certain pour de nombreuses applications industrielles.

Les applications potentielles de ces moteurs dans des secteurs aussi divers que l'automobile, la domotique, la robotique, l'horlogerie ou encore le génie biologique et médical, ont justifié la mise en place de nombreuses actions de recherche au cours des cinq dernières années. Ces actions spécifiques, qui ont reçu le soutien du C.N.R.S. (Centre National de la Recherche Scientifique) et du M.E.N.E.S.R. (Ministère de l'Éducation Nationale, de l'Enseignement Supérieur et de la Recherche), ont conduit à la réalisation de prototypes de laboratoire ainsi qu'au développement de logiciels destinés à leur optimisation $[1,2]$. Les premières réalisations ont révélé des caractéristiques mécaniques exceptionnelles :

- rapport couple/masse élevé,

- couple de maintien à l'arrêt,

- résolution élevée,

- absence de champs magnétiques parasites,

- dynamique de positionnement élevée en régime transitoire,

- absence de bruits audibles de fonctionnement,

- faisabilité de moteurs multi-degrés de liberté.

Le Laboratoire de Mécanique Appliquée R. Chaléat (L.M.A.R.C.) a participé à tous les programmes de recherche nationaux consacrés depuis 1990 aux moteurs et micromoteurs piézoélectriques mono ou multi-degrés de liberté $[3,4]$. Indépendamment de son implication dans le développement de prototypes innovants [5], il a surtout conduit des recherches dans le domaine de la modélisation mécanique des moteurs piézo-électriques à onde progressive, qui représentent, parmi les nombreux dispositifs actuellement étudiés, l'une des voies les plus prometteuses pour garantir prochainement la fabrication de masse de moteurs "fort couple-basse vitesse", sans réducteur mécanique auxiliaire de vitesse.

Les acquis en modélisation de ces cinq dernières années ont permis de démontrer que la quasi- totalité des phénomènes de saturation de puissance des moteurs piézo-électriques à onde progressive ont une origine mécanique [1]. L'approche retenue dès l'origine de nos recherches a 


\section{PRECONTRAINTE AXIALE}

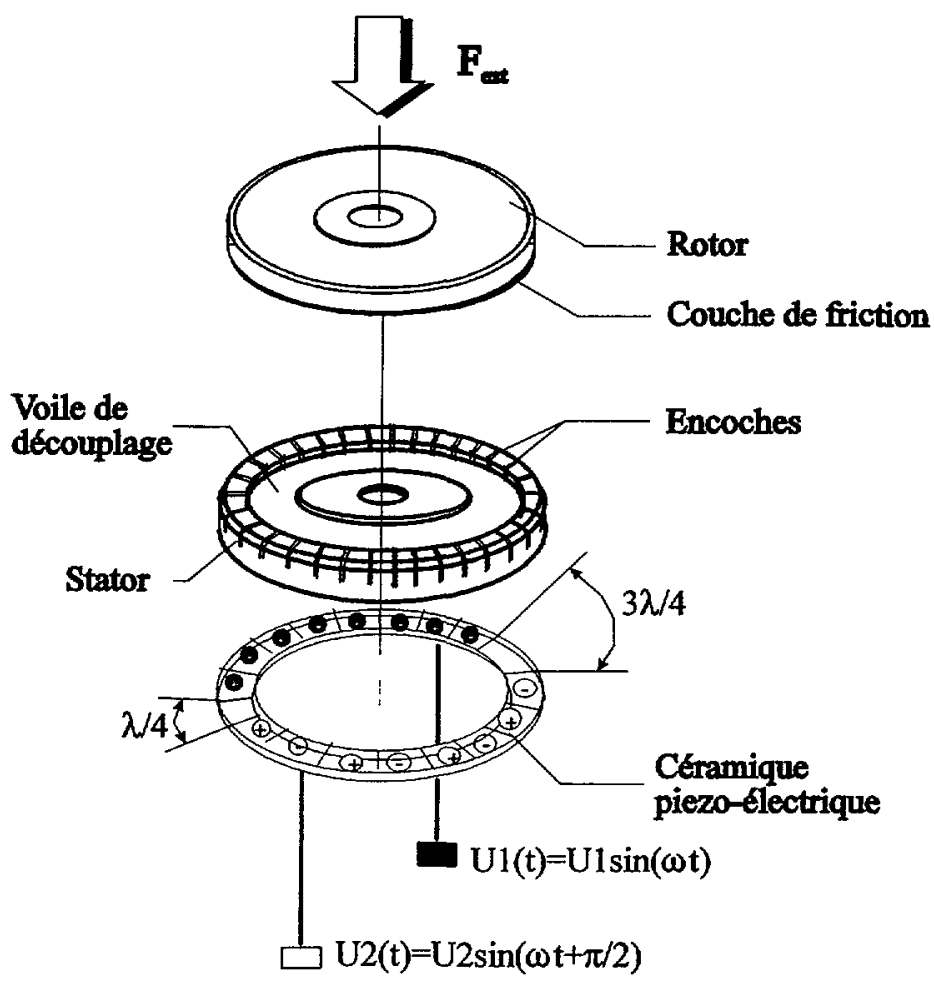

Fig. 1. - Vue éclatée du moteur piézo-électrique rotatif.

[Exploded view of the rotative piezoelectric motor.]

conduit au développement de modèles analytiques prédictifs, reposant sur une analyse purement mécanique du comportement statique et dynamique des moteurs piézo-électriques. Notre activité en modélisation s'est récemment concrétisée par la réalisation de deux logiciels: C.U.B.I.C. (Contact Unilatéral entre Bicouche et Indenteurs Continus) et C.A.S.I.M.M.I.R.E. (Conception Assistée par la Simulation Mécanique des Micromoteurs REsonants), qui ont déjà fait l'objet d'une exploitation dans le secteur industriel.

Cette série de trois articles consacrée au moteur piézo-électrique expose les principes de fonctionnement et les hypothèses qui ont guidé le développement de nos modèles. Elle démontre aussi, validations expérimentales à l'appui, la pertinence de l'approche mécanique initialement retenue pour décrire le comportement global d'un moteur piézo-électrique. Elle indique enfin les limites de validité des hypothèses " $2 \mathrm{D}$ " actuellement retenues par la communauté scientifique internationale en vue d'optimiser le dimensionnement de cette nouvelle génération de moteurs.

\section{Principe de fonctionnement}

L'architecture type d'un moteur à onde progressive est représentée figure 1 dans le cas d'une configuration annulaire. Le stator est excité en vibrations de volume forcées par l'intermédiaire d'un transducteur piézo-électrique collé à sa partie inférieure. La sectorisation du transducteur 
détermine le rang du mode de flexion entretenu sur le stator. Le rang du mode est par ailleurs choisi en fonction des paramètres dimensionnels du moteur, pour garantir l'absence de bruits audibles de fonctionnement (moteurs dits "à ultrasons"). Le rotor est en contact avec la partie supérieure du stator par l'intermédiaire d'un matériau interface. Il est entraîné par frottement en exploitant les déplacements et rotations des sections droites du stator [6]. Le transfert de puissance mécanique à l'arbre moteur nécessite une double transduction d'énergie :

- La première est réalisée à l'interface transducteur-stator par l'intermédiaire d'un joint de colle. Elle exploite l'effet piézo-électrique inverse pour transformer l'énergie électrique provenant de l'alimentation, en énergie mécanique de vibration du stator.

- La seconde s'opère à l'interface stator-rotor par l'intermédiaire du matériau de friction. Les propriétés mécaniques et tribologiques de ce matériau doivent satisfaire la conversion efficace des vibrations de volume du stator en déplacement de corps rigide du rotor. Elles déterminent pour une large part les performances du moteur [7].

L'onde progressive générée sur le stator résulte de la superposition de deux vibrations forcées stationnaires, à une même pulsation $\omega$ voisine d'une pulsation de résonance mécanique $\omega_{k n}$ associée au mode propre de flexion hors plan de rang $(k, n)$ et de multiplicité deux du stator. Les deux formes propres associées à la fréquence propre de pulsation $\omega_{k n}$ présentent par définition un déphasage circonférentiel de $\lambda / 4$ [8], où $\lambda$ désigne la longueur d'onde de la vibration mécanique. L'une des formes propres est excitée par une électrode $E_{1}$ alimentée par la tension électrique $u_{1}(t)=U_{1} \sin \omega t$. L'autre forme propre est excitée par la deuxième électrode $E_{2}$ alimentée par le signal $u_{2}(t)=U_{2} \sin (\omega t+\pi / 2)$. $U_{1}$ et $U_{2}$ désignent respectivement les amplitudes crêtes des signaux $u_{1}(t)$ et $u_{2}(t)$ fonctions de la variable temps : $t$. Les polarisations initiales des céramiques (repérées $\oplus$, sur la Fig. 1) permettent de satisfaire la forme propre choisie.

La quadrature de phase dans l'espace des deux formes propres (déphasage de $\lambda / 4$ ) implique la symétrie mécanique circulaire du stator $[9,10]$. Elle suppose également l'axisymétrie du dispositif de raccordement stator-bâti et du chargement extérieur imposé par l'intermédiaire de la précontrainte axiale exercée sur le rotor. C'est la raison pour laquelle les moteurs à onde progressive sont par définition des moteurs rotatifs. On notera cependant que quelques projets émergent actuellement des laboratoires, dans le but d'étendre les principes précédents à des configurations géométriques variées $[11,12]$.

\section{Hypothèses du modèle de conversion d'énergie à l'interface stator/rotor}

La modélisation des moteurs piézo-électriques à onde progressive intéresse aujourd'hui de nombreux laboratoires et industriels. La diversité des phénomènes physiques mis en jeu a conduit au développement de nombreux modèles, reposant notamment sur l'identification et l'exploitation de schémas électromécaniques équivalents [13-15]. Ces modèles phénoménologiques permettent d'accéder à l'ensemble des caractéristiques électromécaniques des vibromoteurs. Ils nécessitent toutefois l'acquisition expérimentale de paramètres fondamentaux (facteur de force, admittance motionnelle) sur des prototypes de laboratoire réalisés au préalable et s'avèrent peu prédictifs dès qu'une modification sensible des paramètres nominaux est introduite. Les modèles théoriques basés sur des approches numériques de type éléments finis constituent par conséquent une alternative intéressante [16]. Ils requièrent néanmoins des moyens matériels et humains importants, parfois incompatibles avec les exigences de fonctionnement des bureaux d'études et nécessitent des temps de travail importants pour la saisie du problème, le calcul et enfin le traitement et la visualisation graphique des résultats. L'expérience montre que ces approches 
numériques deviennent rapidement inexploitables au stade de l'avant-projet, lorsqu'il s'agit d'évaluer un grand nombre de paramètres mécaniques et géométriques.

Les modèles purement analytiques (explicites ou implicites) sont alors un prolongement naturel des développements numériques initialement entrepris. Ils sont encore cependant pratiquement inexistants dans la littérature actuelle consacrée aux vibromoteurs, en raison sans doute des mécanismes fortement non linéaires, impliqués dans la conversion d'énergie mécanique aux interfaces stator-rotor.

Les travaux menés depuis 1991 au LMARC ont donc pour objectif le développement de modèles analytiques prédictifs, destinés à la conception et l'optimisation mécanique des vibromoteurs, et permettant de simuler la globalité des mécanismes impliqués dans leur fonctionnement $[6,17]$. Ce premier article résume les résultats obtenus dans le domaine de la modélisation cinématique et dynamique de l'interface stator-rotor. Il démontre que l'approche conduite au L.M.A.R.C. aboutit à la simulation de l'ensemble des caractéristiques motrices à vide et en charge du moteur à onde progressive.

\section{Mécanisme d'entraînement du rotor}

4.1. Modélisation Cinématique et dynamique du STATOR. - La génération d'une onde de volume progressive impose le respect de contraintes portant sur la périodicité mécanique circulaire de la structure statorique du moteur [10]. Ces contraintes privilégient le développement actuel de moteurs rotatifs, notamment dans la configuration annulaire définie par la figure 1. L'équation aux dérivées partielles des vibrations hors plan du stator s'écrit, conformément à la théorie classique des plaques circulaires [9] :

$$
\frac{E_{1} h_{1}^{3}}{12\left(1-\nu_{1}^{2}\right)} \nabla^{4} w(r, \theta, t)+\rho_{1} h_{1} \frac{\partial^{2} w(r, \theta, t)}{\partial t^{2}}=0
$$

où $E_{1}, \nu_{1}$ et $\rho_{1}$ désignent respectivement le module de Young, le coefficient de Poisson et la masse volumique du matériau statorique, $h_{1}$ l'épaisseur de la plaque et $\nabla^{4}=\nabla^{2} \nabla^{2}$ avec :

$$
\nabla^{2}=\frac{\partial^{2}}{\partial r^{2}}+\frac{1}{r} \frac{\partial}{\partial r}+\frac{1}{r^{2}} \frac{\partial^{2}}{\partial \theta^{2}}, \quad \text { opérateur de Laplace exprimé en coordonnées polaires. }
$$

Lorsque la symétrie circulaire de la plaque est satisfaite, il existe des solutions de la forme :

$$
w(r, \theta, t)=\xi_{k n}(r) \cos k \theta \sin \omega_{k n} t,
$$

où $k$ et $n$ désignent respectivement les nombres de diamètres nodaux et de cercles de nœuds dans les directions circonférentielle et radiale de la plaque.

La fonction de Bessel $\xi_{k n}(r)$ et la pulsation propre $\omega_{k n}$ sont déterminées à partir de la résolution du problème aux valeurs propres. Lorsque $n=0$, les fréquences propres $\omega_{k n}$ sont de multiplicité deux. Il existe dans ce cas, deux fonctions propres linéairement indépendantes :

$$
\left\{\begin{array}{l}
W^{(1)}(r, \theta)=\xi_{k n}(r) \cos k \theta \\
W^{(2)}(r, \theta)=\xi_{k n}(r) \cos \left(k \theta+\frac{\pi}{2}\right) .
\end{array}\right.
$$

On montre ainsi qu'à une fréquence propre de multiplicité deux du stator, on peut superposer deux vibrations libres d'équation :

$$
\left\{\begin{array}{l}
W^{(1)}(r, \theta, t)=A^{(1)} \xi_{k n}(r) \cos k \theta \sin \omega_{k n} t \\
W^{(2)}(r, \theta, t)=-A^{(2)} \xi_{k n}(r) \sin k \theta \cos \left(\omega_{k n} t+\phi\right),
\end{array}\right.
$$




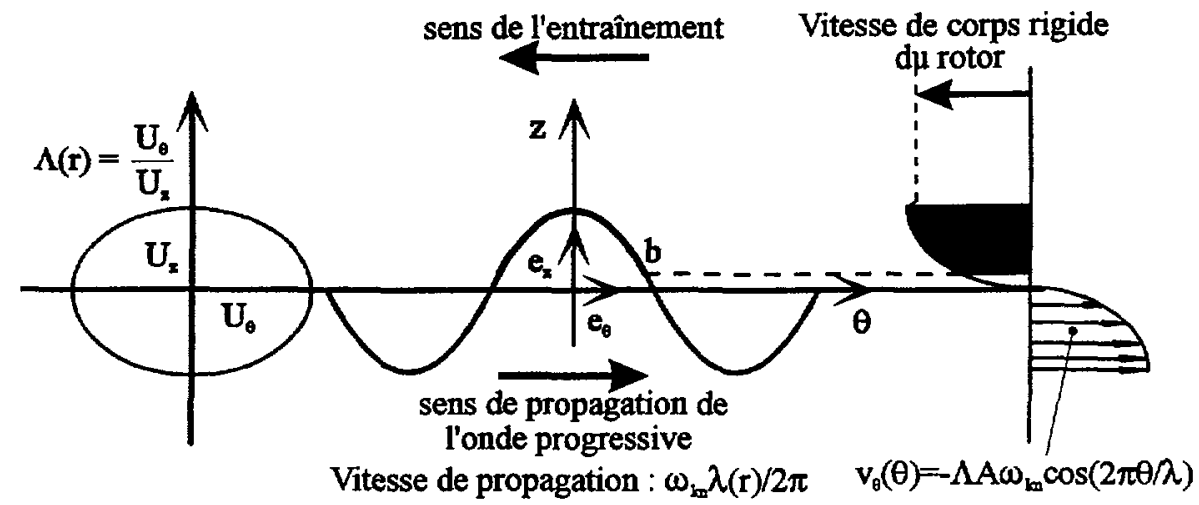

Fig. 2. - Champ des vitesses linéaires d'entraînement.

[Field of the driving linear speeds.]

où $A^{(1)}, A^{(2)}$ et $\phi$ sont des constantes d'intégration qui dépendent des conditions initiales. Si par ailleurs deux vibrations libres ont même amplitude et sont en quadrature de phase dans le temps, la combinaison des deux modes propres génère une onde progressive d'équation :

$$
w(r, \theta, t)=A \xi_{k n}(r) \sin \left(\omega_{k n} t-k \theta\right)
$$

Les paramètres du mouvement d'un point $\mathrm{P}$ de la surface active du stator, situé à une distance $H_{1}$ du plan moyen, s'écrivent selon l'hypothèse de Kirchhoff :

$$
\begin{aligned}
& \mathbf{u}_{\mathbf{P}}(r, \theta, t)=\mathbf{u}_{z}+\mathbf{u}_{r}+\mathbf{u}_{\theta}=w(r, \theta, t) \mathbf{e}_{z}-H_{1} \frac{\partial W}{\partial r}(r, \theta, t) \mathbf{e}_{r}-\frac{H_{1}}{r} \frac{\partial \dot{w}}{\partial \theta}(r, \theta, t) \mathbf{e}_{\theta} \\
= & A\left[\xi_{k n}(r) \sin \left(\omega_{k n} t-k \theta\right) \mathbf{e}_{z}-H_{1} \frac{\partial \xi_{k n}(r)}{\partial r} \sin \left(\omega_{k n} t-k \theta\right) \mathbf{e}_{r}+\frac{k H_{1}}{r} \xi_{k n}(r) \cos \left(\omega_{k n} t-k \theta\right) \mathbf{e}_{\theta}\right] .
\end{aligned}
$$

Par conséquent, le point $\mathrm{P}$ décrit dans le plan $(\theta, z)$ une trajectoire elliptique que l'on peut exprimer sous la forme :

$$
\left(\frac{u_{\theta}}{\Lambda(r) A \xi_{k n}(r)}\right)^{2}+\left(\frac{u_{z}}{A \xi_{k n}(r)}\right)^{2}=1
$$

où $\Lambda(r)=\frac{U_{\theta}(r)}{U_{z}(r)}=\frac{k H_{1}}{r}=\frac{2 \pi H_{1}}{\lambda(r)}$ est un coefficient adimensionnel qui détermine l'allure de la trajectoire; $U_{\theta}$ et $U_{z}$ désignant respectivement les déplacements crêtes dans les directions $\theta$ et $z ; \lambda$ définit la longueur d'onde de la vibration mécanique.

On peut ainsi définir le champ des vitesses d'entraînement des particules statoriques impliquées dans l'interface (Fig. 2), en fonction de l'abscisse circonférentielle $\theta$ sous la forme :

$$
v_{\theta}(r, \theta)=-\Lambda(r) A \xi_{k n}(r) \omega_{k n} \cos \left(\frac{2 \pi r \theta}{\lambda(r)}\right)
$$


Étant donné que les rayons intérieur $\left(R_{0}\right)$ et extérieur $\left(R_{1}\right)$ de l'interface sont voisins, on considérera par la suite que l'amplitude et la longueur d'onde de la vibration mécanique sont indépendantes de $r$ dans le mécanisme d'entraînement du rotor. On supposera par conséquent :

$$
\left\{\begin{array}{l}
\lambda(r)=\lambda=\frac{\pi\left(R_{0}+R_{1}\right)}{k}, \\
A \xi_{k n}(r)=\bar{A}=A \frac{1}{\left(R_{1}-R_{0}\right)} \int_{R_{0}}^{R_{1}} \xi_{k n}(r) \mathrm{d} r
\end{array}\right.
$$

ce qui implique : $\Lambda(r)=\Lambda=2 \pi H_{1} / \lambda$.

Le champ des vitesses linéaires des particules statoriques impliquées dans l'entraînement s'écrit dans ces conditions :

$$
V_{\theta}(\theta)=-\Lambda \bar{A} \omega_{k n} \cos \left(\frac{2 \pi \theta}{\lambda}\right)
$$

où $\theta$ représente désormais l'abscisse curviligne relative au rayon de contact moyen de l'interface : $\left(R_{1}+R_{0}\right) / 2$.

4.2. Tribologie du CONTACT À L'INTERfaCe STATOR-Rotor. - La conversion d'énergie mécanique à l'interface stator-rotor implique la recherche de couches de friction adaptées, capables de convertir sous fort chargement axial les vibrations de volume du stator en mouvement de corps rigide du rotor. La transformation d'énergie par friction requiert une étude phénoménologique des comportements tribologiques associés aux diverses familles de couches de matériaux interfaces exploitables (polymères, céramiques, métaux...).

La prédiction des performances mécaniques des vibromoteurs nécessite notamment l'évaluation des variations du coefficient de frottement dynamique à l'interface couche-stator, en fonction des vitesses de glissement particulaires représentées figure 3 . Une étude tribologique du contact stator-rotor a donc été réalisée en collaboration avec le Laboratoire de Technologie des Surfaces (L.T.S.) de l'École Centrale de Lyon (France), dans le cas des mécanismes de contact impliquant des couches de polymères (matériaux les plus utilisés à l'heure actuelle). La figure 4 indique les fluctuations du coefficient de frottement enregistrées en fonction des vitesses de glissement relatives des particules statoriques et rotoriques. Les acquisitions ont été réalisées en valeur locale, à partir d'un contact bille-plan sur un tribomètre spécifique permettant de restituer les conditions de fonctionnement d'un vibromoteur type. Les enregistrements pratiqués dans une plage de vitesse compatible avec les champs de vitesses de glissement observés sur la majorité des moteurs, rendent compte de fluctuations importantes de $\mu_{\mathrm{d}}$ au voisinage des conditions de fonctionnement statique définies par $\mu_{\mathrm{s}}$.

Les résultats expérimentaux observés sur le cas générique du moteur SHINSEI USR 60 ont fait l'objet d'un lissage mathématique représenté figure 5 . Ce lissage a par la suite été introduit dans l'algorithme de calcul des performances motrices sous la forme d'une loi de friction ayant pour expression :

$$
\mu_{\mathrm{d}}(\theta)=\operatorname{sgn}\left(v_{\mathrm{glis}}(\theta)\right)\left[\mu_{\mathrm{Max}}+\left(\mu_{\min }-\mu_{\mathrm{Max}}\right) \mathrm{e}^{\frac{-\left|v_{\mathrm{gl} \mathrm{I}_{\mathrm{s}}}(\theta)\right|}{\tau}}\right],
$$

où $v_{\text {glis }}(\theta)=v_{\theta}(\theta)-V_{\text {rotor }}$.

Pour des considérations pratiques, nous adopterons par la suite les notations suivantes:

$$
\left\{\begin{array}{lll}
\mu_{\mathrm{d}}(\theta)=\mu_{\mathrm{d}}^{+}(\theta) & \text { si } & v_{\text {glis }}(\theta)>0 \\
\mu_{\mathrm{d}}(\theta)=\mu_{\mathrm{d}}^{-}(\theta) & \text { si } & v_{\text {glis }}(\theta)<0 .
\end{array}\right.
$$




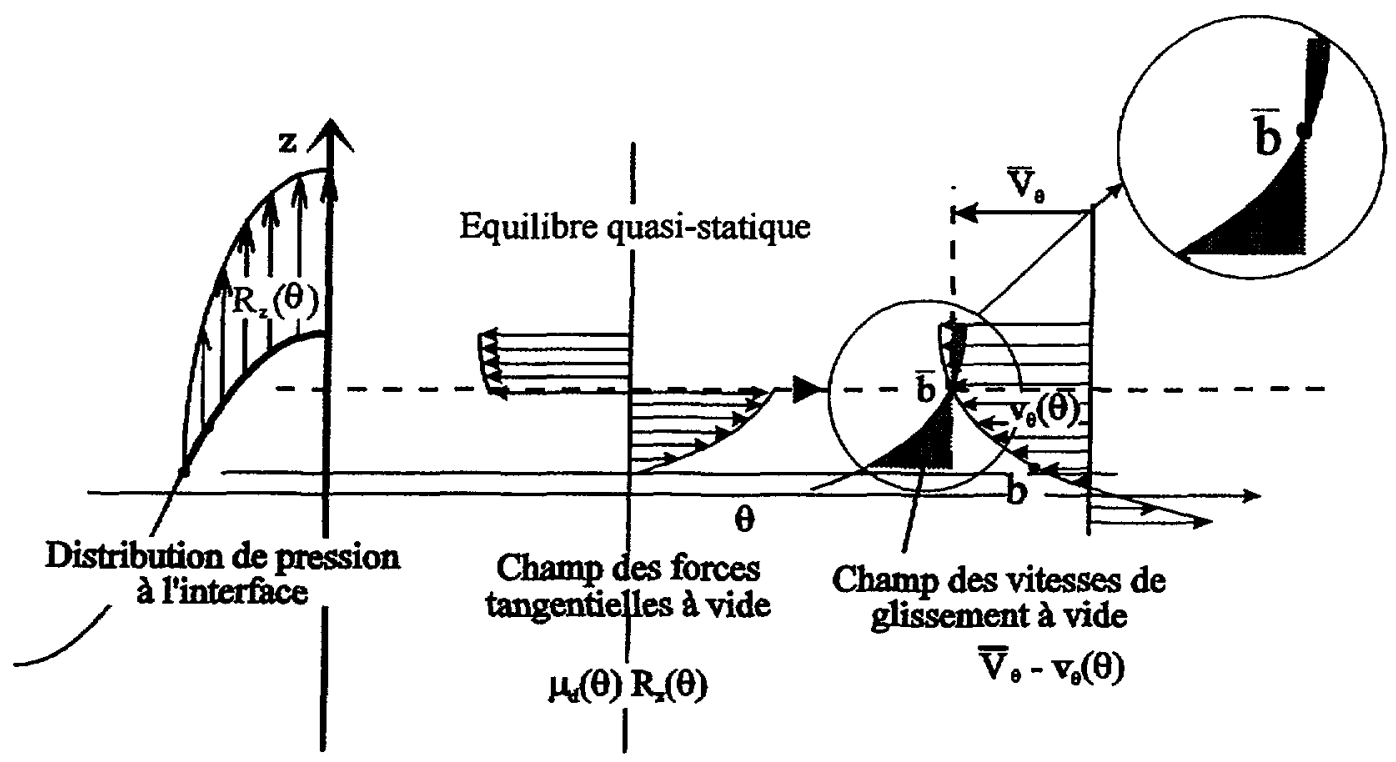

Fig. 3. - Champ des forces tangentielles d'entraînement à vide.

[Field of the driving tangential forces in the no-loading case.]

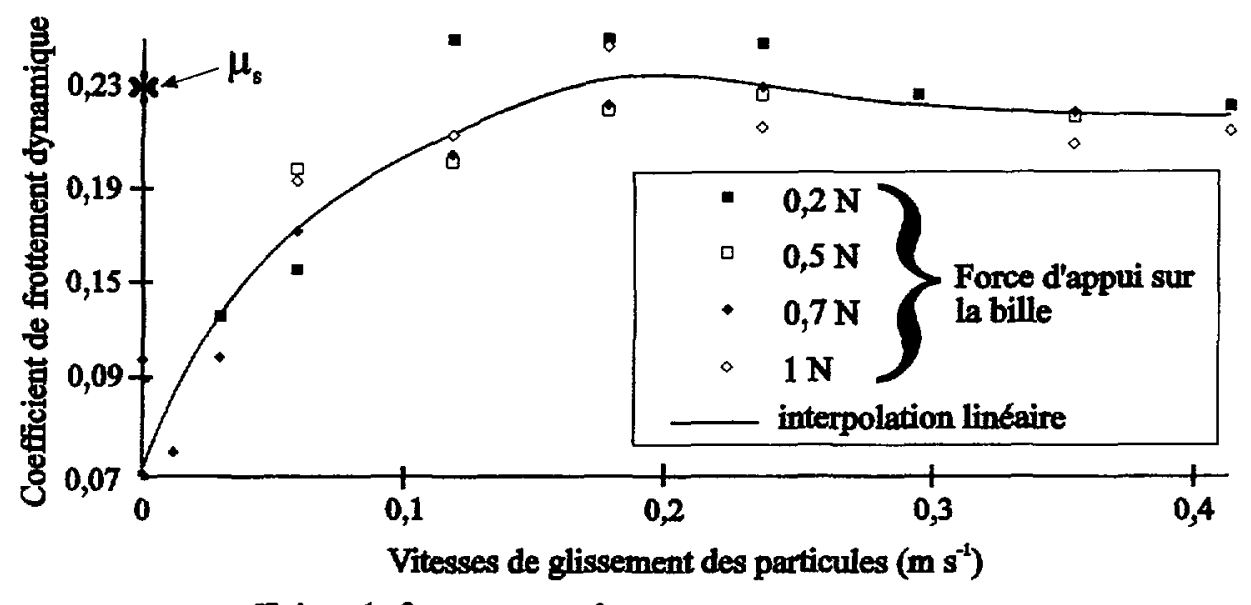

$\mu_{s}$ coefficient de frottement statique

Fig. 4. - Évaluation expérimentale du coefficient de frottement dynamique en fonction des vitesses de glissement.

[Dynamic friction coefficient variations with respect to the sliding speeds.]

4.3. VITESSE LINÉAIRE DE CORPS RIGIDE COMMUNIQUÉE AU ROTOR. - La vitesse de corps rigide communiquée au rotor est fonction du taux de contact $K(K=2 b / \lambda)$ à l'interface statorrotor (Fig. 2). La vitesse angulaire de l'arbre moteur dépend par conséquent de la précontrainte axiale $F_{\text {ext }}$ exercée sur l'arbre moteur. Elle est maximale en l'absence de couple résistant 


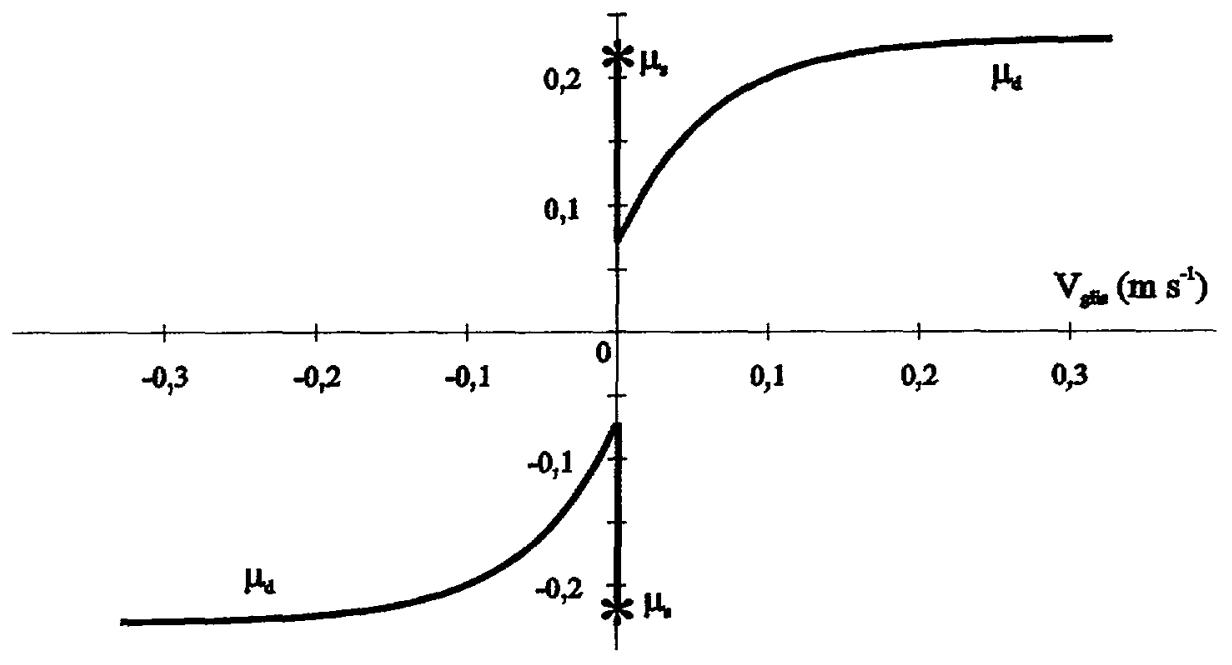

Fig. 5. - Loi de variation du coefficient de frottement introduite dans le modèle de l'interface.

[Analytical fitting of the dynamic friction coefficient introduced in the interface model.]

$\left(\Gamma_{\mathrm{r}}=0\right)$. On supposera par la suite que la vitesse linéaire maximale de corps rigide du rotor $\bar{V}_{\theta}$ (quand $\Gamma_{\mathrm{r}}=0$ ), s'identifie à la vitesse vibratoire tangentielle de la particule d'abscisse $\theta=\bar{b}$ localisée à la surface du stator vibrant (Fig. 3).

L'équilibre quasi-statique du rotor dans la direction $\theta$ permet de définir le lieu de $\bar{b}$ (lorsque $\Gamma_{\mathrm{r}}=0$ ), à partir de l'équilibre des forces tangentielles (Fig. 3 ) induites par friction à l'interface stator-rotor.

$\mathrm{Si}$ on suppose que le coefficient de frottement dynamique $\mu_{\mathrm{d}}$ est indifférent aux vitesses de glissement à l'interface, le lieu de $\bar{b}$ est défini par l'égalité :

$$
\int_{0}^{\bar{b}}\left[R_{z}(\theta)\right]_{F_{\text {ext }}} \mathrm{d} \theta-\int_{\bar{b}}^{b}\left[R_{z}(\theta)\right]_{F_{\text {ext }}} \mathrm{d} \theta=0,
$$

où $R_{z}(\theta)$ représente la distribution de pression statique normale sur l'interface délimitée par la borne $b$, dont le lieu dépend du chargement axial $F_{\text {ext }}$.

On notera que la symétrie du problème autorise l'analyse des mécanismes de contact et d'entraînement sur une demi-longueur d'onde. De ce fait, la plupart des calculs se rapportera à une demie-interface de contact délimitée par 0 point de symétrie et $b$.

Par ailleurs, il résulte de l'étude tribologique initiale du contact que le calcul de $\bar{b}$ doit être, compte tenu de l'équation (9) réécrit sous la forme:

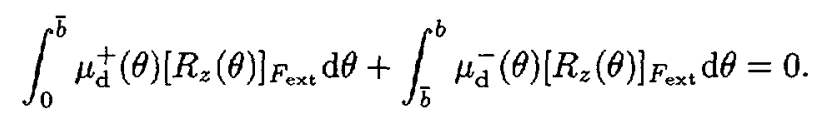

Le calcul de la borne $\bar{b}$ permet d'exprimer, compte tenu de (8), la vitesse linéaire maximale de corps rigide du rotor (en $r=\frac{R_{0}+R_{1}}{2}$ ) sous la forme :

$$
\bar{V}_{\theta}=v_{\theta}(\theta=\bar{b})=-\Lambda \bar{A} \omega_{k n} \cos \left(\frac{2 \pi \bar{b}}{\lambda}\right) \text {. }
$$


L'évaluation de la vitesse théorique à vide du rotor $\left(\Gamma_{\mathrm{r}}=0\right)$ impose par conséquent la modélisation statique initiale du contact, dans le but d'évaluer la limite $b$ du contact, la distribution de pressions $R_{z}(\theta)$ et la borne $\bar{b}$ en fonction des paramètres de définition mécaniques et géométriques de l'interface.

\section{Modélisation des caractéristiques motrices à vide et en charge}

5.1. DÉcouplage deS MOdèles NORMAL ET TANGEnTiel DE L'INTERface. - Les résultats précédents ont été exploités pour l'évaluation théorique des performances mécaniques des moteurs à onde progressive. Un algorithme permettant le calcul analy tique des caractéristiques en charge ainsi que l'optimisation de la précontrainte axiale exercée sur l'arbre moteur a été développé sur la base des résultats précédents. Le principe opératoire de cet algorithme repose sur l'hypothèse du découplage des modèles normal et tangentiel de l'interface. Plus précisément, on supposera dans ce qui suit, que l'application de sollicitations mécaniques extérieures dans la direction circonférentielle de l'interface (couple résistant) ne perturbe pas la symétrie initiale de la géométrie du contact sous chargement normal. Tiang et Saka [18] ont effectivement démontré que la géométrie du contact est pratiquement indifférente au chargement tangentiel lorsque le coefficient de friction à l'interface stator-rotor est inférieur ou égal à 0,3 (valeur typiquement supérieure à celles observées aux interfaces des moteurs à ultrasons).

Les calculs suivants supposent par ailleurs une amplitude vibratoire crête constante et impliquent par conséquent la conception d'une alimentation électronique auxiliaire, autorisant l'asservissement fréquentiel du moteur en fonction des chargements extérieurs successivement imposés [19].

Lorsque ces contraintes pratiques sont satisfaites, l'expérience montre que le champ des vitesses vibratoires dans la direction circonférentielle de l'interface stator-rotor est indifférent aux chargements mécaniques imposés à l'arbre moteur.

5.2. Distributions THÉORIQUES DES RÉACTIONS DE CONTACT NORMALES. - L'évaluation théorique des caractéristiques motrices des vibromoteurs à onde progressive impose l'intégration de fonctions continues, donnant la description des réactions de contact normales et tangentielles sur l'intervalle de définition de l'interface $[0, b]$. L'obtention des réactions nodales calculées numériquement impose par conséquent une interpolation afin de garantir la continuité des profils de pression à intégrer. L'interprétation approfondie des simulations a permis de démontrer une correspondance géométrique liant les profils de réactions normales au profil de l'indenteur, quelles que soient les précontraintes axiales successivement imposées à l'arbre moteur. Pour chaque valeur du paramètre $F_{\text {ext }}$, une fonction continue $R_{z}(\theta)$ donnant une approximation satisfaisante des réactions nodales peut être recherchée à partir d'un polynôme du 3ème degré exprimé sous la forme :

$$
\left[R_{\mathrm{z}}(\theta)\right]_{F_{\mathrm{ext}}}=(\theta-b)\left(a^{(1)} \theta^{2}+a^{(2)} \theta+a^{(3)}\right) .
$$

L'identification des constantes $a^{(1)}, a^{(2)}$ et $a^{(3)}$ de l'équation (13), qui impose par ailleurs l'absence de réactions hors de la limite $b$ de l'interface, suppose respectivement l'équilibre statique du rotor dans la direction hors plan $z$; la symétrie des profils de réactions et la 
correspondance liant la géométrie de l'indenteur à la distribution $R_{z}(\theta)$ :

$$
\left\{\begin{array}{l}
\int_{0}^{b} R_{z}(\theta) \mathrm{d} \theta=\frac{F_{\text {ext }}}{2 k} \\
{\left[\frac{\partial R_{z}(\theta)}{\partial \theta}\right]_{\theta=0}=0} \\
{\left[\frac{\partial^{2} R_{z}(\theta)}{\partial^{2} \theta}\right]_{\theta=\lambda / 4}=0}
\end{array}\right.
$$

5.3. ForCes tANGentielles théoriques COMMUNiquées aU ROTOR. - Le principe d'entraînement des moteurs à onde progressive repose sur la conversion, par friction, des vibrations de volume entretenues sur le stator, en déplacements de corps rigide du rotor. Les lois classiques du frottement de Coulomb permettent, compte tenu des hypothèses décrites au paragraphe 5.1, de calculer le champ des forces tangentielles d'entraînement du rotor. Ce champ de forces dépend linéairement de la distribution normale $R_{z}(\theta)$ ainsi que de la valeur particulaire algébrique du coefficient de friction dynamique $\mu_{\mathrm{d}}(\theta)$ (Éq. (9)). Le signe des forces tangentielles d'entrâ̂nement dépend par conséquent du signe des vitesses de glissement des particules matérielles à l'interface stator-rotor.

Lorsque aucune charge n'est imposée dans la direction circonférentielle $\theta$ de l'interface (moteur à vide : $\Gamma_{\mathrm{r}}=0$ ), le champ des vitesses d'entraînement communiqué au rotor satisfait la description schématique de la figure 3 , et le lieu de la particule $\vec{b}$, se déduit de l'écriture de l'équilibre quasi-statique du rotor (11).

Par conséquent, les particules matérielles situées à la surface du stator :

- entraînent le rotor lorsque $\theta \in[0, \bar{b}]$ et $\Gamma_{\mathrm{r}}=0$,

- s'opposent à l'entraînement du rotor lorsque $\theta \in[\vec{b}, b]$ et $\Gamma_{\mathbf{r}}=0$.

5.4. Vitesse angulaire MAXIMALE DE L'ARBRE MOTEuR. - Les calculs précédents permettent d'exprimer la vitesse angulaire maximale à vide $\Omega_{\mathrm{v}}$ de l'arbre moteur sous la forme :

$$
\Omega_{\mathrm{v}}\left(F_{\mathrm{ext}}\right)=\frac{2 \bar{V}_{\theta}}{R_{1}+R_{0}}=-\frac{2 \Lambda \bar{A} \omega_{k n}}{R_{1}+R_{0}} \cos \left(\frac{2 \pi \bar{b}}{\lambda}\right)
$$

Cette vitesse, qui dépend de la précontrainte axiale exercée sur l'arbre moteur, est négative parce que (4) suppose une propagation de l'onde progressive dans la direction $\theta$ positive.

5.5. Caractéristiques théoriques Couple-Vitesse en CHARge. - En imposant la décrémentation de $\Omega_{\mathrm{c}}$. vitesse angulaire de l'arbre moteur chargé par un couple résistant $\Gamma_{\mathrm{r}}$, on peut identifier la localisation de la borne $\bar{b}_{\mathrm{c}}$ définie au paragraphe 5.3 sous la forme :

$$
\bar{b}_{c}=\frac{\lambda}{2 \pi} \arccos \left[\Omega_{c} \frac{\left(R_{0}+R_{1}\right)}{2 \Lambda \bar{A} \omega_{k n}}\right] \quad \text { et } \quad \bar{b}_{c} \leq b
$$

Deux cas distincts peuvent alors apparaître selon le niveau de chargement axial exercé sur le rotor :

a) Taux de contact $K>50 \%$

Dans ce premier cas, la relation (16) autorise le calcul de $\vec{b}_{c}$ jusqu'au blocage définitif de l'arbre moteur $\left(\Omega_{\mathrm{c}}=0\right)$ et le couple moteur $\Gamma$ disponible sur l'arbre satisfait l'équation suivante :

$$
\Gamma=k\left(R_{0}+R_{1}\right)\left\{\int_{0}^{\vec{b}_{\mathrm{c}}} \mu_{\mathrm{d}}^{+}(\theta)\left[R_{z}(\theta)\right]_{F_{\text {ext }}} \mathrm{d} \theta+\int_{\tilde{b}_{\mathrm{c}}}^{b} \mu_{\mathrm{d}}^{-}(\theta)\left[R_{z}(\theta)\right]_{F_{\text {ext }}} \mathrm{d} \theta\right\} .
$$


b) Taux de contact $K<50 \%$

Dans ce deuxième cas, le couple moteur $\Gamma$ disponible vérifie la relation (17) dans l'intervalle de définition :

$$
v_{\theta}(\theta=b)=\Omega_{\mathrm{c}}\left(\frac{R_{0}+R_{1}}{2}\right) \leq \bar{V}_{\theta} \leq \Omega_{\mathrm{v}}\left(\frac{R_{0}+R_{1}}{2}\right)
$$

où $\bar{V}_{\theta}$ est la vitesse linéaire de corps rigide du rotor à $r=\left(R_{0}+R_{1}\right) / 2$.

La figure 6 donne l'interprétation géométrique correspondante.

Lorsque le couple résistant $\Gamma_{\mathrm{r}}$ freine le rotor à une vitesse linéaire (en $\left.r=\left(R_{0}+R_{1}\right) / 2\right) \bar{V}_{\theta}<v_{\theta}$ $(\theta=b)$, la borne $\bar{b}_{\mathrm{c}}$ n'a plus de signification physique. Dans ce cas, l'évolution du couple moteur (Fig. 7) est due à l'augmentation du coefficient de frottement dynamique $\mu_{\mathrm{d}}$ consécutive à l'augmentation des vitesses de glissement (Fig. 5) :

$$
\Gamma=k\left(R_{0}+R_{1}\right) \int_{0}^{b} \mu_{\mathrm{d}}^{+}(\theta)\left[R_{z}(\theta)\right]_{F_{\mathrm{ext}}} \mathrm{d} \theta
$$

5.6. Couple statique de maintien à l'arrêt. - Les lois classiques du frottement de Coulomb permettent par ailleurs d'évaluer très simplement le couple statique de maintien à l'arrêt disponible sur l'arbre moteur. Lorsque l'alimentation est interrompue, la pression statique unitaire à l'interface stator-rotor vérifie l'égalité :

$$
p_{\mathrm{s}}=\frac{F_{\mathrm{ext}}}{\pi\left(R_{1}^{2}-R_{0}^{2}\right)} .
$$

Le moment de frottement exercé par un anneau élémentaire de rayon $R$ et de largeur $\mathrm{d} R \mathrm{~s}$ 'écrit par ailleurs :

$$
\mathrm{d} \Gamma_{\mathrm{S}}=\mu_{\mathrm{S}} r \mathrm{~d} F_{\mathrm{ext}}=2 \mu_{\mathrm{S}} p_{\mathrm{S}} \pi r^{2} \mathrm{~d} r
$$

où : $\mu_{\mathrm{s}}$ et $\mathrm{d} F_{\text {ext }}$ définissent respectivement le coefficient de frottement statique à l'interface stator-rotor et la force normale exercée par l'anneau élémentaire.

Le couple statique de maintien à l'arrêt $\Gamma_{\mathrm{s}}$ vérifie dans ces conditions :

$$
\Gamma_{\mathrm{s}}=2 \mu_{\mathrm{s}} p_{\mathrm{s}} \pi \int_{R_{0}}^{R_{1}} r^{2} \mathrm{~d} r=\frac{2}{3} \mu_{\mathrm{s}} F_{\text {ext }} \frac{R_{1}^{3}-R_{0}^{3}}{R_{1}^{2}-R_{0}^{2}}
$$

5.7. Pertes mécaniques dans la direction Circonferentielle de l'interface. Le principe de fonctionnement des vibromoteurs piézo-électriques implique l'existence de glissements relatifs entre les particules statoriques et rotoriques impliquées dans l'interface. Ces glissements sont à l'origine de pertes mécaniques par friction. Les calculs précédents autorisent l'évaluation de ces pertes dans la direction circonférentielle $\theta$ de l'interface. En supposant une hypothèse de glissement généralisé entre toutes les particules de l'interface (à l'exception de la particule d'abscisse $\theta=\bar{b}_{\mathrm{c}}$ ), et en imposant à nouveau la décrémentation de $\Omega_{\mathrm{c}}$, on peut exprimer, compte tenu de (9), les vitesses de glissement $v_{\mathrm{glis}}(\theta)$ en fonction de $\theta$ sous la forme :

$$
v_{\text {glis }}(\theta)=\Lambda \bar{A} \omega_{k n} \cos \left(\frac{2 \pi \theta}{\lambda}\right)-\frac{R_{0}+R_{1}}{2} \Omega_{\mathrm{c}} .
$$




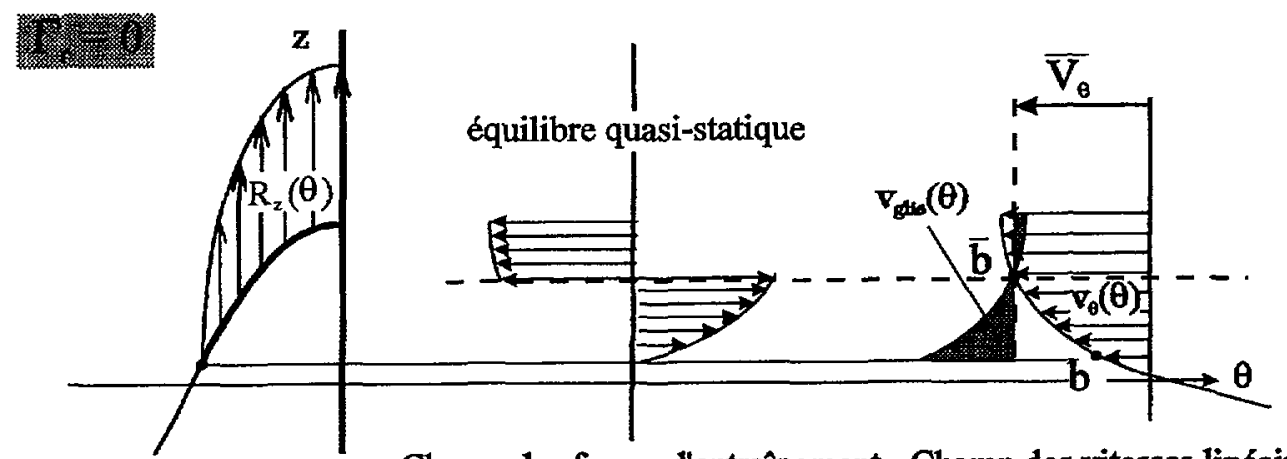

Distribution de pression Champ des forces d'entraînement Champ des vitesses linéaires à l'intenface $\mu_{d}\left(v_{d t b}\right) R_{d}(\theta)$ $v(\theta)=\Lambda \bar{A} \omega \cos (2 \pi \theta / \lambda)$

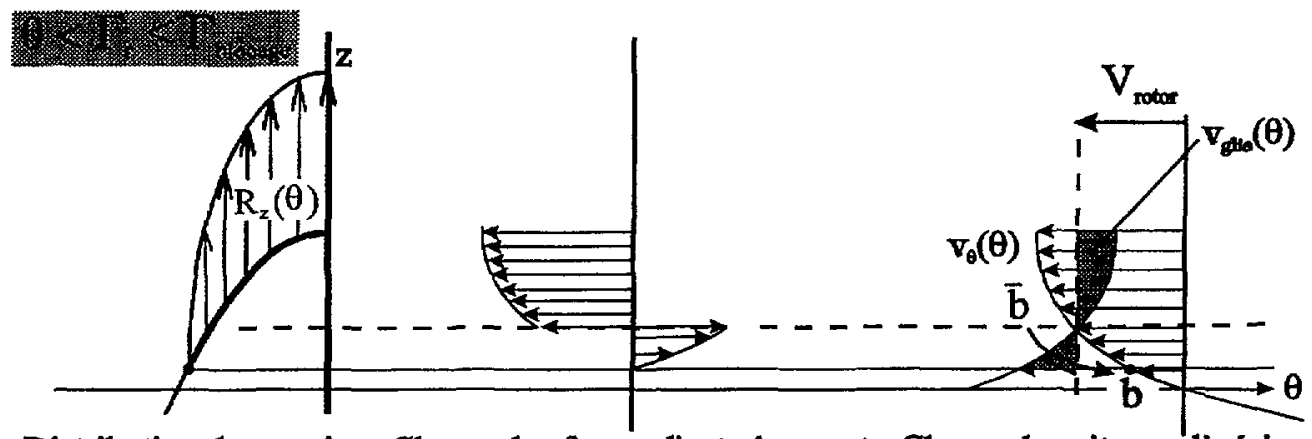

Distribution de pression Champ des forces d'entraînement Champ des vitesses linéaires Al linterface $\mu_{d}\left(v_{\text {ditu }}\right) R_{\alpha}(\theta)$ $v(\theta)=\Lambda \bar{A} \omega \cos (2 \pi \omega / \lambda)$

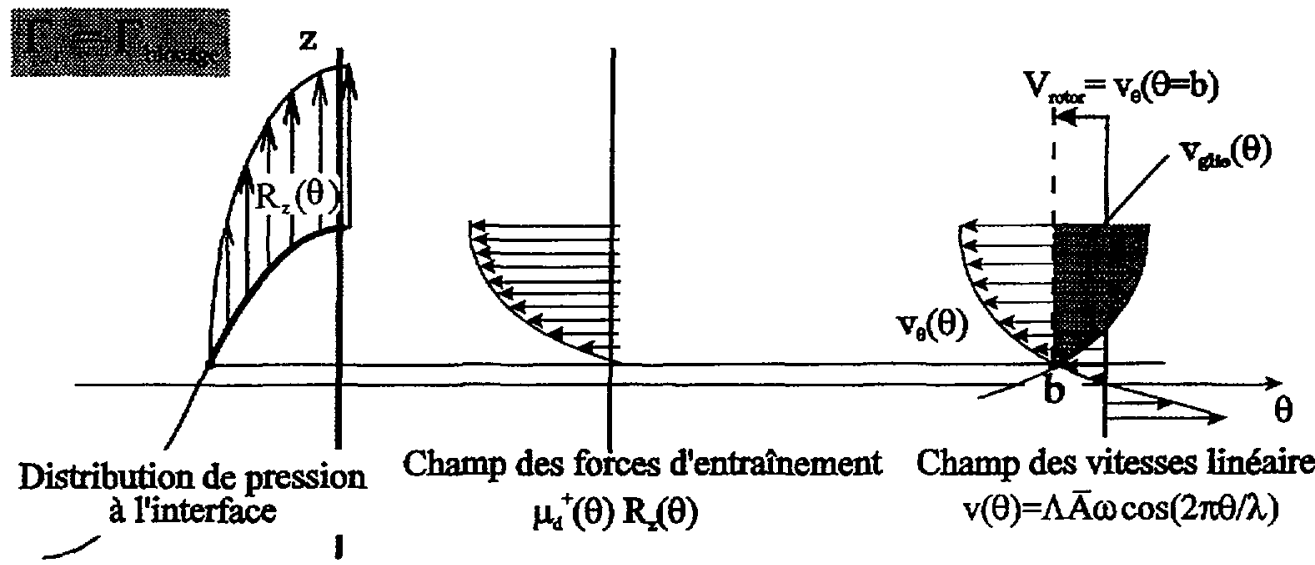

Fig. 6. - Interprétation graphique du comportement en charge.

[Driving mechanism of the rotor.] 


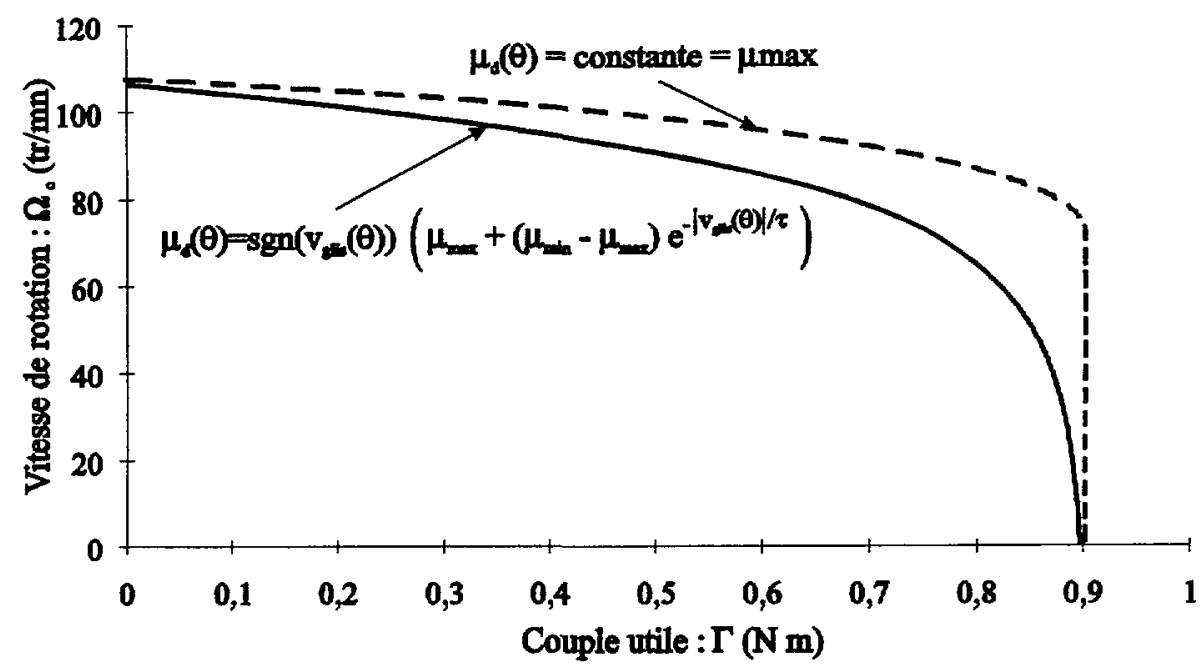

Fig. 7. - Caractéristiques couple-vitesse théoriques du moteur SHINSEI USR 60 - Influence des lois de frottement.

[Theoretical loading characteristics of the SHINSEI USR 60 motor.]

Les pertes mécaniques à l'interface stator-rotor sont alors définies par :

$$
P_{\text {glis }}=2 k \Lambda \bar{A} \omega_{k n}\left\{\begin{array}{l}
\int_{0}^{\bar{b}_{\mathrm{c}}} \mu_{\mathrm{d}}^{+}(\theta)\left[R_{z}(\theta)\right]_{F_{\text {ext }}}\left(\cos \left(\frac{2 \pi \theta}{\lambda}\right)-\frac{R_{0}+R_{1}}{2 \Lambda \bar{A} \omega_{k n}} \Omega_{\mathrm{c}}\right) \mathrm{d} \theta \\
+\int_{\bar{b}_{\mathrm{c}}}^{b} \mu_{\mathrm{d}}^{-}(\theta)\left[R_{z}(\theta)\right]_{F_{\text {ext }}}\left(\cos \left(\frac{2 \pi \theta}{\lambda}\right)-\frac{R_{0}+R_{1}}{2 \Lambda \bar{A} \omega_{k n}} \Omega_{\mathrm{c}}\right) \mathrm{d} \theta
\end{array}\right\}
$$

$\operatorname{si} \Omega_{\mathrm{c}}\left(\frac{R_{0}+R_{1}}{2}\right)>V_{\theta}(\theta=b)$.

Lorsque la vitesse angulaire $\Omega_{\mathrm{c}}$ chute de sorte que $\Omega_{\mathrm{c}}\left(\frac{R_{0}+R_{1}}{2}\right)<V_{\theta}(\theta=b)$ l'équation (23) devient :

$$
P_{\mathrm{glis}}=2 k \Lambda \bar{A} \omega_{k n}\left\{\int_{0}^{b} \mu_{\mathrm{d}}^{+}(\theta)\left[R_{z}(\theta)\right]_{F_{\mathrm{ext}}}\left(\cos \left(\frac{2 \pi \theta}{\lambda}\right)-\frac{R_{0}+R_{1}}{2 \Lambda \bar{A} \omega_{k n}} \Omega_{\mathrm{c}}\right) \mathrm{d} \theta\right\}
$$

5.8. Chargement axial théorique optimal. - Les calculs précédents autorisent l'évaluation théorique de la puissance mécanique communiquée à l'arbre moteur en fonction des paramètres de définition de l'interface stator-rotor. La précontrainte axiale optimale $F_{\text {ext }}^{\text {opt }}$ est alors définie par :

$$
\left[\frac{\partial\left(\Gamma \Omega_{c}\right)_{\mathrm{Max}}}{\partial F_{\mathrm{ext}}}\right]_{F_{\mathrm{ext}}^{\mathrm{opt}}}=0
$$

Le schéma synoptique de la figure 8 donne la description de l'algorithme d'optimisation du chargement axial extérieur. 


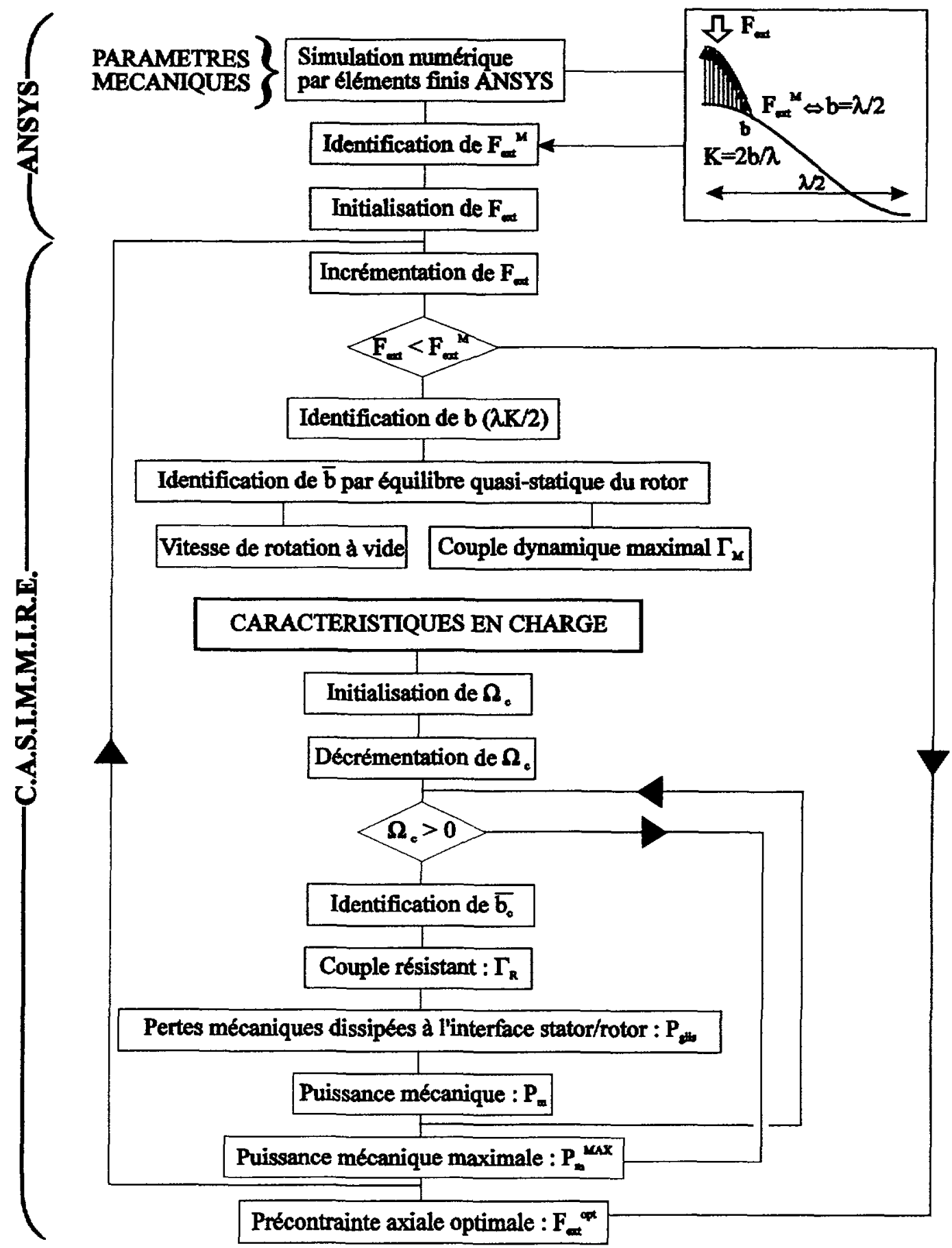

Fig. 8. - Schéma synoptique de l'algorithme exploité dans le logiciel C.A.S.I.M.M.I.R.E. [Algorithm developed in the C.A.S.I.M.M.I.R.E. software.] 

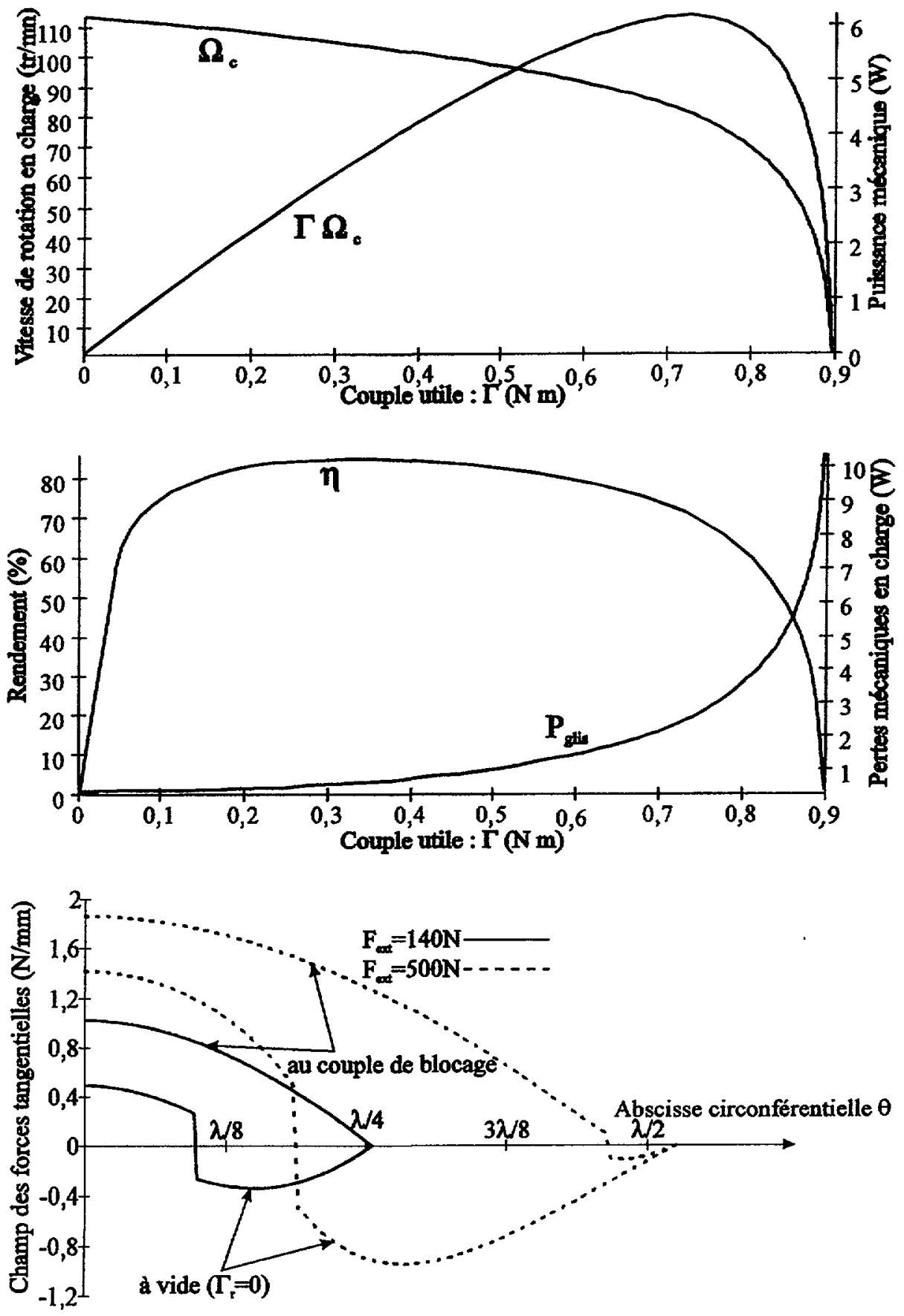

Fig. 9. - Caractéristiques théoriques nominales en charge du moteur SHINSEI USR $60\left(F_{\text {ext }}=140 \mathrm{~N}\right)$ (visualisation graphique à partir du logiciel C.A.S.I.M.M.I.R.E.).

[Theoretical characteristics of the SHINSEI USR 60 motor at the operating preload.] 

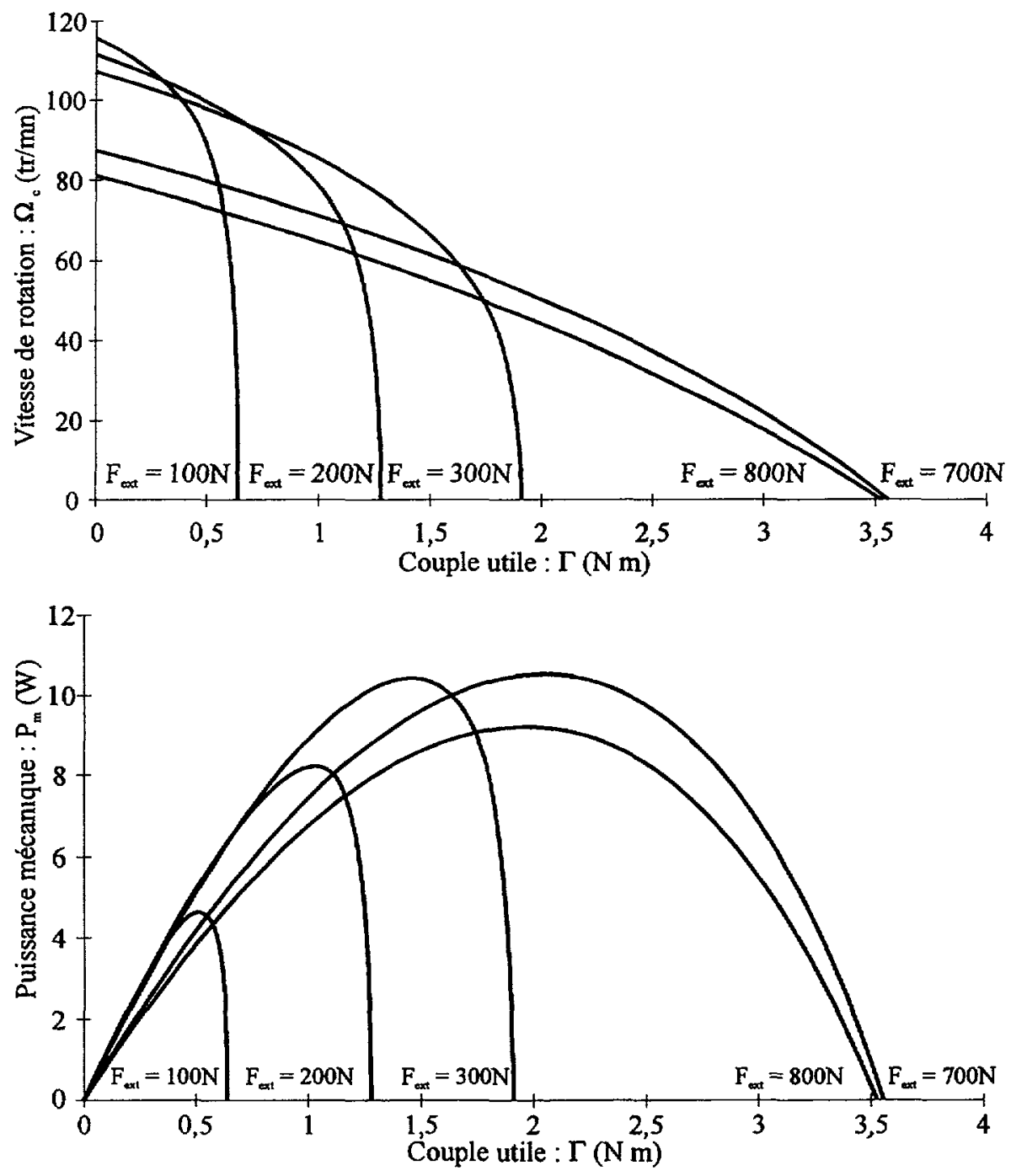

Fig. 10. - Caractéristiques théoriques en charge pour différents niveaux de précontrainte axiale (visualisation graphique à partir du logiciel C.A.S.I.M.M.I.R.E.).

[Theoretical loading characteristics for different axial preload steps.]

5.9. INTERPRÉtATION DES SAISIES GRAPHIQUES. - La modélisation théorique de la conversion d'énergie mécanique à l'interface stator-rotor nous a récemment conduits au développement d'un deuxième logiciel : C.A.S.I.M.M.I.R.E. (Conception Assistée par SImulation Mécanique des MIcromoteurs RÉsonants). Ce logiciel intègre un interface graphique et a été spécifiquement développé pour la conception et l'optimisation mécanique des moteurs et micromoteurs piézo-électriques à onde progressive. La formulation analytique du mécanisme de conversion des vibrations de volume du stator en déplacement de corps rigide du rotor est particulièrement bien adaptée à la résolution des problèmes de dimensionnement au stade de l'avant-projet. 

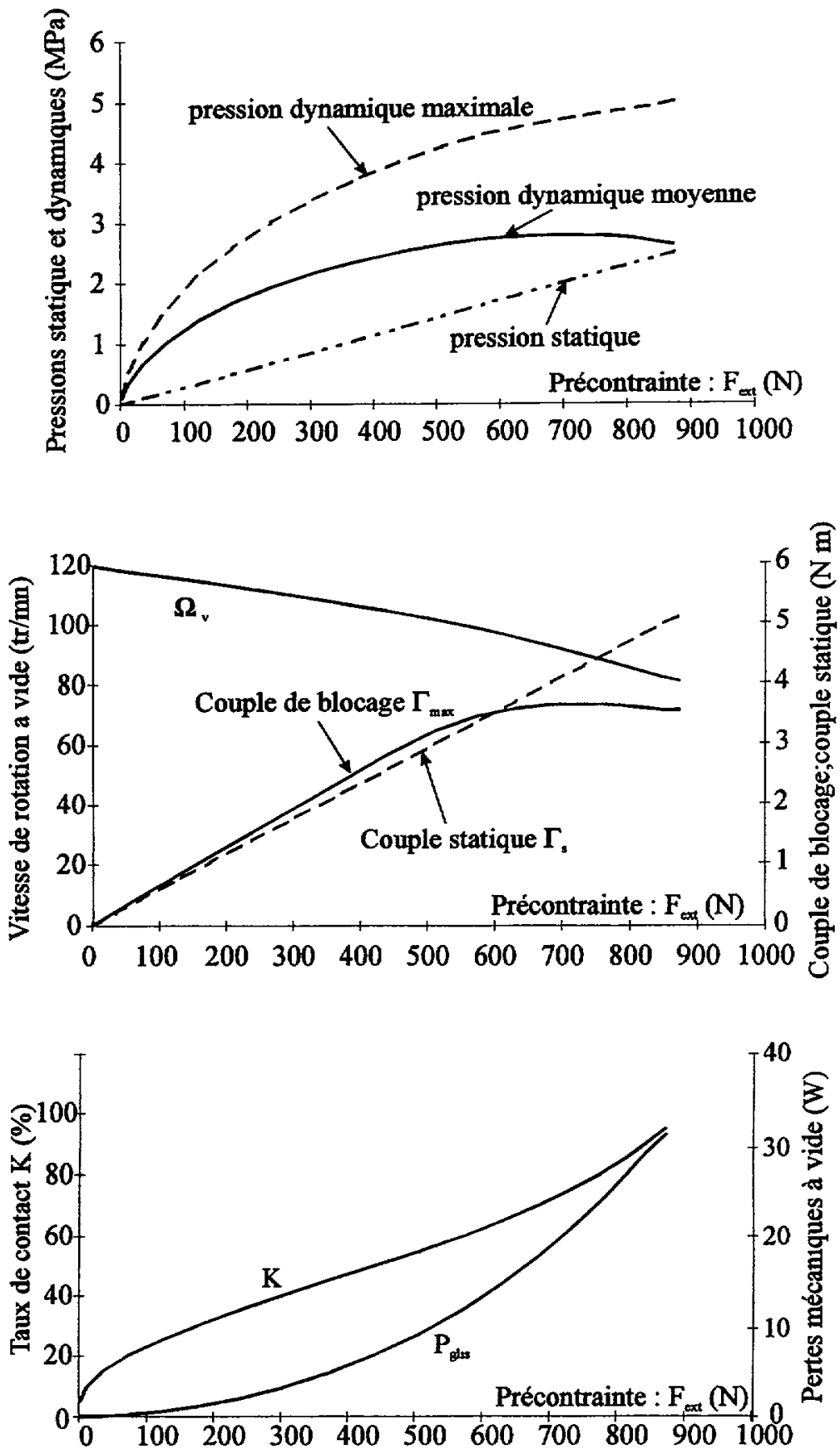

Fig. 11. - Caractéristiques limites en fonction de la charge axiale (visualisation graphique à partir du logiciel C.A.S.I.M.M.I.R.E.).

[Mechanical characteristics as a function of the axial preload.] 


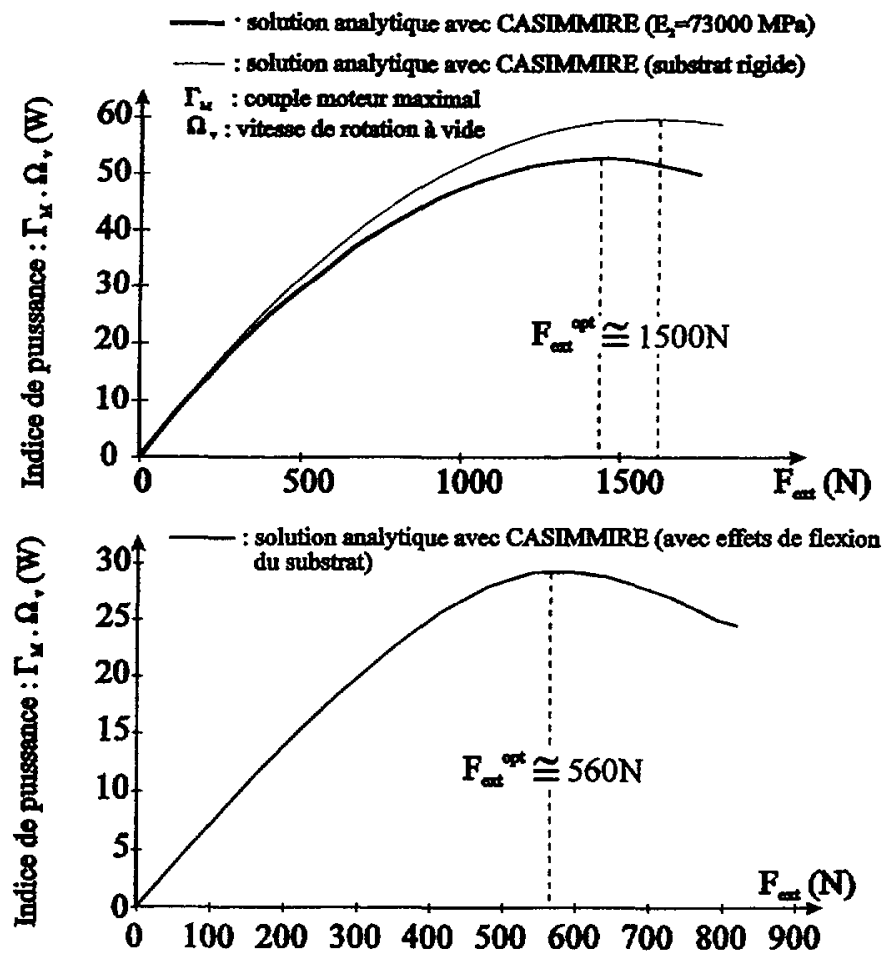

Fig. 12. - Évaluation théorique de la précontrainte axiale optimale par C.A.S.I.M.M.I.R.E. [Theoretical calculation of the optimal axial preload performed by C.A.S.I.M.M.I.R.E.]

Le logiciel C.A.S.I.M.M.I.R.E. calcule et visualise toutes les caractéristiques mécaniques d'un moteur vibrant en moins d'une minute sur micro-ordinateur PC, ce qui en fait un outil de simulation privilégié pour les bureaux d'études des PMI.

À titre indicatif, les caractéristiques théoriques du moteur de référence SHINSEI USR 60 (Figs. 9, 10, 11 et 12), ont été obtenues après quelques dizaines de secondes de calcul. Ces informations fournissent l'essentiel des données utiles d'une part à l'optimisation mécanique du moteur et d'autre part à la synthèse de couches de friction pertinentes (polymères chargés, céramiques...) capables de convertir efficacement l'énergie mécanique à l'interface stator-rotor.

La figure 9 indique les résultats des simulations du moteur SHINSEI USR 60 en charge. Les caractéristiques ont été calculées pour la valeur nominale de la précontrainte axiale choisie par le constructeur. On constate que le rendement mécanique à l'interface stator/rotor est voisin de $80 \%$ lorsque le moteur fonctionne à son régime nominal. Les champs des forces tangentielles d'entraînement sont représentés à titre indicatif pour deux valeurs distinctes de précontrainte axiale $\left(F_{\text {ext }}=140 \mathrm{~N}\right.$ et $500 \mathrm{~N}$ ), car ils fournissent des informations essentielles pour la synthèse de couches de friction.

La figure 10 donne une évaluation du potentiel mécanique du moteur SHINSEI USR 60. L'interprétation des courbes indique que les performances motrices sont extrêmement sensibles à la précontrainte axiale exercée sur l'arbre moteur. Il est clair qu'indépendamment des problèmes d'usure liés au comportement tribologique de l'interface, une optimisation mécanique du moteur pourrait permettre un gain significatif de puissance (et notamment de couple). On 
Observation : la variation d'épaisseur de la couche (1) traduit l'influence des déformations de contacts ; la déflexion de la couche (2) traduit le mécanisme de flexion circonférentielle du rotor dans la configuration $F_{\text {ext }}=140 \mathrm{~N}$.

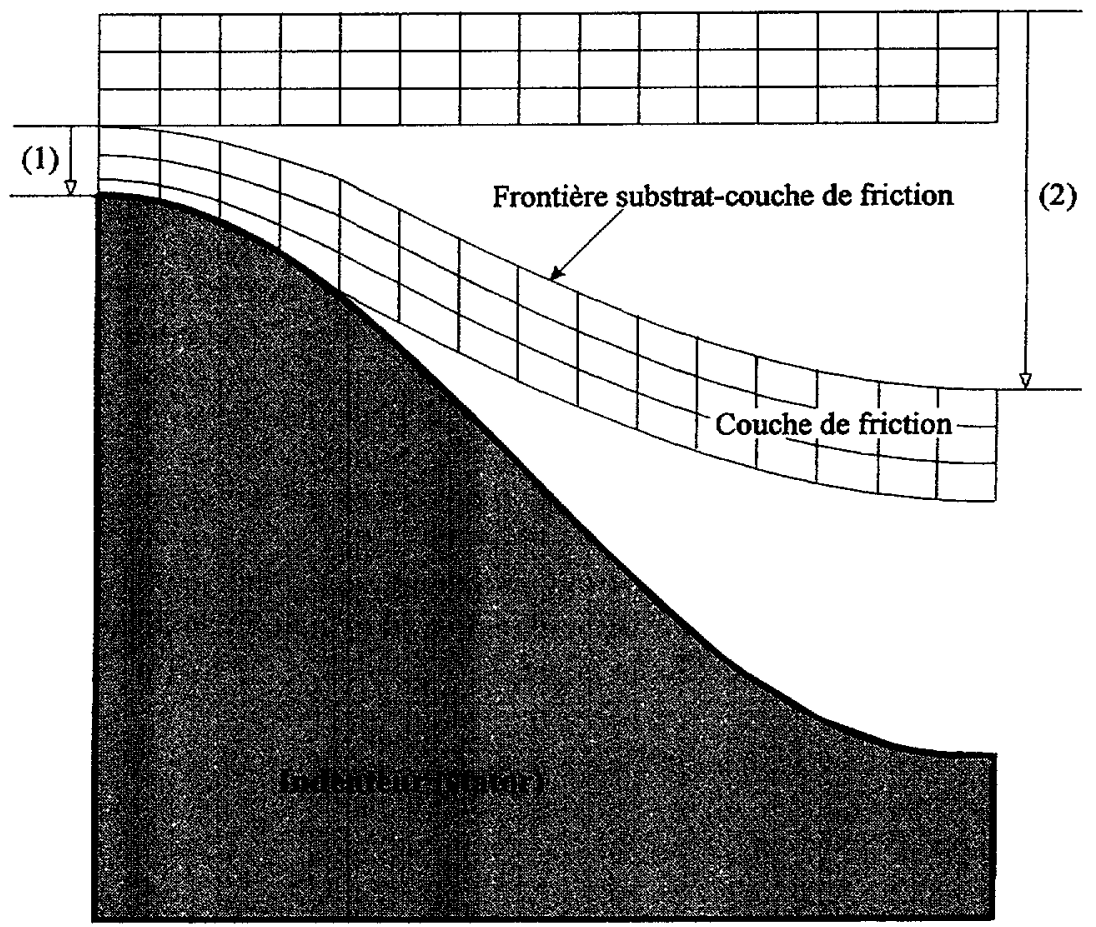

Fig. 13. - Visualisation par ANSYS des déplacements induits respectivement par les déformations de contact et par la flexion circonférentielle du rotor.

[Numerical modeling of the interface (ANSYS): visualization of the contact deformations and the bending effects.]

constate qu'au-delà d'un niveau excessif de chargement axial, les caractéristiques couple-vitesse saturent (saturation du couple de blocage dynamique), même si par ailleurs, le couple statique de maintien à l'arrêt continue d'évoluer linéairement en fonction de $F_{\text {ext }}$ (Fig. 11).

La figure 11 visualise les lois de comportement du moteur en fonction de $F_{\text {ext }}$. Indépendamment des caractéristiques limites $\Omega_{\mathrm{v}}, \Gamma_{\mathrm{Max}}$ et $\Gamma_{\mathrm{s}}$ qui intéressent directement les industriels, le logiciel C.A.S.I.M.M.I.R.E. donne des indications utiles au concepteur (pertes mécaniques et pressions à l'interface stator rotor ; les pressions dynamiques maximale et moyenne sont relatives à l'interface stator/rotor lorsque le stator est effectivement excité en vibrations de volume), dans la phase de dimensionnement du prototype.

La figure 12 donne enfin des estimations théoriques de la précontrainte axiale optimale qui devrait être exercée sur l'arbre moteur du SHINSEI USR 60. Ces estimations résultent de l'exploitation de l'algorithme d'optimisation décrit sur la figure 8. Elles ont été calculées et visualisées par C.A.S.I.M.M.I.R.E. en fonction de diverses hypothèses (substrat rigide ; substrat massif déformable semi-infini ; substrat fléchissant dans le plan $(z, \theta)$ ) afin de souligner notamment l'influence des effets de flexion à haut niveau de chargement axial (Fig. 13) (effets systématiquement négligés dans les publications scientifiques actuellement consacrées aux moteurs à ultrasons $[20,21])$. 


\section{Conclusion et perspectives}

Les modèles présentés dans ce premier article autorisent l'évaluation théorique des performances mécaniques des moteurs à onde progressive. La description analytique du mécanisme de conversion d'énergie à l'interface stator/rotor est particulièrement adaptée pour la conception et l'optimisation de prototypes au stade de l'avant-projet.

Toutefois, compte tenu de la complexité des mécanismes non linéaires agissant au niveau du contact stator/rotor, nous avons eu recours au calcul numérique pour accéder aux entrées $\mathrm{du}$ modèle de conversion d'énergie à l'interface (taux et réactions normales de contact). Cette approche coûteuse en temps de calcul : de l'ordre de quelques heures sur des stations de type H.P. 900715 (temps auxquels il convient d'ajouter la saisie des géométries et des maillages ainsi que les traitements de données toujours délicats avec les logiciels de calcul par éléments finis) nous a ultérieurement conduits aux développements d'algorithmes spécifiques donnant accès, analytiquement, à l'ensemble des paramètres de définition du contact stator/couche de friction/rotor. Ces algorithmes sont présentés dans le deuxième article consacré à la modélisation théorique des moteurs à onde progressive. On verra ultérieurement que les résultats acquis au L.M.A.R.C. ont abouti à un modèle de moteur purement analytique, qui a par ailleurs pu être expérimentalement validé à partir de la caractérisation approfondie de piézomoteurs Japonais de type SHINSEI USR 45 et USR 60.

\section{Bibliographie}

[1] Minotti P., Moteurs Piézo-électriques : Modélisation et Conception Mécanique, Rapport Confidentiel C.N.R.S. (1996).

[2] Le Moal P., Minotti P., Ferreira A. and Cusin P., Design and optimization of batchfabricated rotative piezomotors, SPIE 1996 Symposium on smart structures and materials (San Diego, USA, February 25-29, 1996) pp. 565-575.

[3] Minotti P., Étude et Réalisation d'un Moteur à Ultrasons, Rapport M.E.S.R. (1992).

[4] Minotti P. et Ferreira A., Micromoteurs Piézo-électriques Multi-Degrés de Liberté, Rapport Action Spécifique D.S.P.T. 8 Génie Mécanique (1995).

[5] Buchaillot L., Modélisation Mécanique Théorique des Moteurs Piézo-électriques à Onde Progressive, Thèse de doctorat de l'Université de Franche-Comté (Besançon, 1995).

[6] Minotti P. et Lallement G., Modélisation théorique des moteurs piézo-électriques rotatifs à ondes progressives, Mech. Mach. Theory 29 (1994) 281-292.

[7] Rouchon J.F., Kapsa P., Minotti P., Buchaillot L. et Duffait R., Problèmes de surfaces dans les moteurs à ultrasons, Matériaux et Techniques $\mathrm{n}^{\circ}$ 5-6 (1995) 37-42.

[8] Hagedorn P. and Wallaschek J., Traveling wave ultrasonic motors part 1: Working principles and mathematical modelling of the stator, J. Sound Vibration 115 (1992) 31-46.

[9] Leissa A.W., Vibration of plates, National Aeronautics and Space Administration (Washington, 1969) pp. 344.

[10] Geradin M. et Rixen D., Théorie des vibrations - Applications à la dynamique des structures, Masson (Paris, 1993) pp. 231.

[11] Manceau J.F., Bastien F. and Duffait R., Ultrasonic micromotor stator evaluation, IEEE Ultrasonics Symposium (Cannes, France, 1994).

[12] Minotti P. et El Hami A., Conception et modélisation d'un moteur piézo-électrique linéaire à onde progressive, Mech. Mach. Theory (à paraître). 
[13] Sashida T. and Kenjo T., An introduction to ultrasonic motors, Clarendon Press (Oxford, 1993).

[14] Hirata H. and Ueha S., Characteristics estimation of a traveling wave type ultrasonic motor, IEEE Trans. Ultrason. Ferroelect. Frequency Control 40 (1993) 402-406.

[15] Le Letty R., Conception de moteurs piézo-actifs à l'aide de la modélisation, Thèse de Doctorat (Institut National des Sciences Appliquées, Lyon, 1994).

[16] Maeno T., Tsukimoto T. and Miyake A., Finite-element analysis of the rotor/stator contact in a ring-type ultrasonic motor, IEEE Trans. Ultrason. Ferroelect. Frequency Control 29 (1992) 668-674.

[17] Minotti P., Conception et modélisation de mécanismes - Applications dans le domaine des moteurs piézo-électriques, Mémoire d'Habilitation à Diriger des Recherches (rapport Confidentiel Industrie, 1994).

[18] Tian H. and Saka N., Finite element analysis of an elastic-plastic two-layer half-space: sliding contact, Wear 148 (1991) 261-285.

[19] Minotti P., Buchaillot L. et Ferreira A., Modélisation mécanique théorique et caractérisation des moteurs piézo-électriques à onde progressive (Rapport C.N.R.S. Confidentiel Industrie, 1994).

[20] Hirata $\mathrm{H}$. and Ueha S., Design of a traveling wave type ultrasonic motor, IEEE Trans. Ultrason. Ferroelect. Frequency Control 42 (1995) 225-231.

[21] Hagood N.M. and Mc Farland A.J., Modeling of a piezoelectric rotary ultrasonic motor, IEEE Trans. Ultrason. Ferroelect. Frequency Control 42 (1995) 210-224. 